\title{
ANÁLISIS COMPARADO SOBRE PATRIMONIO CULTURAL INDÍGENA Y CURRÍCULO: AUSTRALIA, CHILE Y SUDÁFRICA
}

\section{Felipe Aravena ${ }^{1}$}

\section{RESUMEN}

En este estudio se busca, a través de casos comparados, analizar cómo es abordado el patrimonio cultural de las comunidades indígenas en el currículo nacional de Australia, Chile y Sudáfrica. Los referentes curriculares analizados son: para Australia, Crosscurriculum priorities: Aboriginal and Torres Strait Islander Histories and Cultures (2015); en el caso sudafricano, National Curriculum Statements: Grades R-12 (2013) y, finalmente, para el caso chileno el Marco curricular lengua indígena $1^{\circ}$ a $6^{\circ}$ básico (2009). El estudio sostiene que es necesario valorar el patrimonio cultural indígena a nivel curricular, aunque el salto cualitativo que deben dar los diseñadores curriculares esté en la generación de estrategias y orientaciones de aprendizaje contextualizadas con estudiantes indígenas y no indígenas. La metodología utilizada es de corte cualitativo de análisis de casos comparados en tres dimensiones: identidad, categorización curricular y conocimiento y aprendizaje indígena. A partir de los resultados se pueden desarrollar proyecciones curriculares acerca del adecuado enfoque del patrimonio cultural indígena, considerando la validación de los conocimientos de estas comunidades como prioridad. Finalmente, se concluye que en los tres casos se ha optado por la mantención de un currículo monocultural integrando parcialidades del patrimonio cultural indígena y obteniendo efectos en los procesos de construcción de identidades, de integración nacional y de diferenciación cultural.

Palabras clave: currículo, conocimiento indígena, identidad, integración nacional.

\section{A COMPARATIVE ANALYSIS ON CULTURAL INDIGENOUS PATRIMONY AND CURRICULUM: AUSTRALIA, CHILE AND SOUTH AFRICA}

\section{ABSTRACT}

The purpose of this article is to conduct a comparative analysis of national curriculum regarding the way indigenous culture patrimony is treated in three different contexts; Australia, Chile and South Africa. The following curriculum is analyzed; Australian case Cross-Curriculum Priorities: Aboriginal and Torres Strait Islander Histories and Cultures (2015); South African case, National Curriculum Statements: Grades R-12 (2013), and finally for the Chilean case Marco Curricular Lengua Indígena $1^{\circ}$ a $6^{\circ}$ básico (2009). This study suggests that it is necessary to value indigenous culture patrimony in the curriculum. The challenge for curriculum developers, however, lies within the creation of strategies and orientations for contextualized

Escuela de Pedagogía, Pontificia Universidad Católica de Valparaíso, Valparaíso, Chile. Contacto: felipe.aravena@pucv.cl 
learning with indigenous and non-indigenous students. The analysis conducted uses a qualitative methodology, comparing the cases along three dimensions: identity, curricular categorization and indigenous content knowledge. The results allow for the development of curriculum projections on an adequate focus of indigenous culture patrimony, considering as a priority, the validation of these communities' knowledge. This study concludes that in all three cases, a monocultural curriculum has been preserved, incorporating a limited vision of indigenous culture patrimony. This option has affected processes of identity building, national integration and cultural differentiation.

Keywords: curriculum, identity, indigenous knowledge, national integration.

\section{Introducción}

Cambios, complejidades y movimientos cada vez más acelerados son los que afectan continuamente al mundo (Marginson, 2010; Rizvi \& Lingard, 2010) y la educación no ha quedado ajena a ellos. En un planeta globalizado, la incertidumbre pareciera ser el común denominador. Dentro de este mundo hiperinterconectado, profundamente complejo y multidimensional, se han creado espacios comunes que tienden a homogeneizar culturalmente la sociedad (Ball, 2012; Carnoy, 1999). En la actualidad, se ha forjado una tendencia educativa de valorar la diversidad como un aspecto clave dentro de los sistemas educativos. La heterogeneidad cultural ha tomado fuerza para presentarse como un aspecto distintivo de las sociedades que debe ser respetado en su esencia, ante la vorágine de los procesos de globalización e internacionalización (Henry, 2010). Bajo esta premisa, se comprende la generación de sistemas, procesos y estructuras más diversas que permitan la inclusión de los que han sido marginados tradicional y globalmente. Esto exhibe una interesante y compleja dialéctica entre lo homogéneo y lo heterogéneo, lo marginal y lo inclusivo, lo global y lo local, lo conocido y lo desconocido, lo ajeno y lo propio.

Dentro de este marco de diversidad e inclusión se inserta el presente estudio, el cual tiene por objetivo analizar, a través de la metodología de estudios de casos comparados, cómo es abordado el patrimonio cultural de comunidades indígenas en el currículo nacional de Australia, Chile y Sudáfrica. Los referentes curriculares analizados son: para Australia, Cross-Curriculum Priorities: Aboriginal and Torres 
Strait Islander Histories and Cultures (The Australian Curriculum, 2015); en el caso sudafricano, National Curriculum Statements: Grades R-12 (Republic of South Africa, 2013) y, finalmente, para el caso chileno se utilizó el Marco curricular lengua indígena $1^{\circ}$ a $6^{\circ}$ básico (Ministerio de Educación de Chile, Mineduc, 2009). La relevancia de este estudio radica en tres aspectos fundamentales: primero, en el reposicionamiento de la integración de comunidades indígenas desde la educación formal basada en estándares nacionales; segundo, en el análisis situado a partir de tres realidades complejas y diversas que atienden a un mismo problema aún no resuelto: cómo integrar de manera significativa el patrimonio cultural indígena dentro del currículo nacional; y, finalmente, como tercer elemento, este estudio da a conocer, a través de un análisis de tensiones educativas, posibles proyecciones de la problematización de la integración/marginalización de las comunidades indígenas a un nivel local, nacional y global.

\section{Patrimonio cultural indígena y currículo}

La cultura es toda creación humana que se entiende dentro de un espacio y tiempo concreto. Geertz (2000) plantea que la complejidad cultural debe ser comprendida en función de la relación que se construye entre el individuo y su ambiente, una interacción altamente simbólica que se establece en una aproximación biológicaambiental. A partir de esta interacción, la cultura se va reeditando, transformando y construyendo dentro de procesos cada vez más complejos en la medida en que se van fundando modos de pensar, lenguaje, símbolos, artefactos y creencias, entre otros elementos. Así, la cultura se presenta como un complejo entramado de relaciones sociales, económicas, políticas e ideológicas (Simpson, 1997).

La cultura se expresa en elementos tangibles (observables) y no tangibles (invisibles), lo que ha sido denominado como patrimonio material e inmaterial, respectivamente (Geertz, 2000; Simpson, 1997). En los primeros, el paisaje natural, arquitectura y objetos forman parte del patrimonio tangible de una comunidad, mientras que en los segundos, las creencias, costumbres y ritos se exhiben como patrimonios culturales no tangibles. Este estudio centrará su atención en ambos (aunque en términos teórico-epistemológicos sea útil hacer 
la distinción entre ellos), ya que es imprescindible considerar ambos elementos como parte de un complejo cultural más amplio (Geertz, 2000), especialmente cuando se analizan culturas desde un todo, sin focalizar la atención en la especificidad y densidad cultural.

Es así como el concepto de patrimonio cultural ha sido discutido desde diferentes perspectivas. En efecto, para Coombe (2001) este incluye las herencias generadas en el pasado, historias y mitos que terminan por direccionar el destino de la comunidad. Kuprecht (2012) señala que el patrimonio cultural crea memoria colectiva y sentido de pertenencia en y para un grupo social determinado. Asimismo, Mcmillan (2013) enfatiza que el patrimonio cultural se hace cargo de la identidad del pueblo, puesto que entrega la posibilidad de conservar y alimentar memorias colectivas a través del entendimiento de un mundo compartido. Patrimonio cultural e identidad son dos conceptos que confluyen a través de una unificación de la historia de la comunidad (García, 2007). En este estudio, el patrimonio cultural será entendido como toda herencia cultural, tangible y no tangible, compartida por un grupo social que se hace acreedor de una misma memoria colectiva, identidad y pasado común.

En los últimos treinta años se ha puesto énfasis en la relación entre patrimonio cultural y currículo (Leguina y Baquedano, 2000). Múltiples han sido los estudios que sitúan el currículo con ideas de reproducción de patrones culturales y más aún con la idea de identidad y patrimonio cultural (Birch, 2010). Se entiende que el currículo no solamente es un documento oficial, sino que es fundamentalmente praxis (Scott, 2008). Lo anterior debido a que reconoce la posibilidad de influenciar y modificar de forma poderosa las prácticas pedagógicas, los conceptos de aprendizaje y enseñanza, así como también la idea de educación e identidad nacional (Birch, 2010; Wang, 2015). Esto lo confirma García (2007) señalando que el patrimonio cultural ha sido utilizado como una estrategia fundamental para unificar y crear símbolos nacionales, unificando así la diversidad cultural. De forma que si en el currículo se ha establecido la idea de patrimonio cultural es debido a la necesidad imperiosa de generar las condiciones para establecer un sentido de pertenencia histórica. 
Más precisamente, la relación entre currículo y patrimonio cultural indígena es compleja. Esto, porque existen problemas epistemológicos en la construcción del conocimiento establecido desde parámetros curriculares (Quilaqueo, Fernández y Quintriqueo, 2010). El currículo centralizado, emanado de los organismos gubernamentales, tiende a ser monocultural, desvalorizando o suprimiendo desde la desconfianza la validez del conocimiento y saber popular indígena (Merino, 2006), lo que hace preferir una monopolización del conocimiento y deslegitimando la posibilidad de un cruce relacional intercultural en las lógicas de aprendizaje y racionalidad de un mundo y otro. Por consiguiente, se opta por unificar la diversidad cultural haciendo necesaria la construcción de un currículo sostenido en una educación intercultural más pertinente y contextualizada a la realidad de las diferentes comunidades indígenas (Merino, 2006; Quilaqueo et al., 2010; Quintrineo y McGinity, 2009).

Leguina y Baquedano (2000) manifiestan que el patrimonio cultural está asociado a lo antiguo, lo pasado, "lo no vivo", por lo cual es difícil aproximarse a él ya que suele percibirse como inamovible $y$, por tanto, intocable. No obstante, el patrimonio cultural se puede revitalizar acercándolo a los estudiantes y a la sociedad mediante una reconfiguración en el currículo nacional (Nakata, 2011). Así, se ha instalado la idea de que este puede aportar en la restauración, protección y valoración del patrimonio cultural indígena, siendo esto último una responsabilidad sustentada en una voluntad macropolítica que implica influenciar el proceso de construcción de la memoria colectiva, en nuevas formas de relaciones socioculturales y en prácticas de poder concentradas en crear un sentido de unidad histórica.

Las comunidades indígenas y su patrimonio cultural frecuentemente han sido clasificadas como culturas exclusivas y diferenciadas dentro de los currículos nacionales (Nakata, 2011; Rogers, 2012; Simpson, 1997). Son presentadas como una colectividad diferenciada, que si bien se desea integrar, permanece marginada a través de una diferenciación cultural. En unos casos es una marginación explícita, pero en la mayoría de ellos implícita. 
Abramovich (2006) enfatiza que las comunidades indígenas no han sido capaces de autoconcebirse como actores demandantes en el proceso de diseño e implementación curricular, porque aún se sirven del asistencialismo educativo. Sin embargo, en los últimos años ha existido una disposición proclive a reconocer el valor de las comunidades indígenas dentro del currículo como parte fundante de un pasado compartido (Putnam, David, Bernard \& Jerome, 2011). Con ello, se abre la oportunidad de encontrarse y dialogar culturalmente con un mundo indígena desconocido, diferente y por tanto interesante desde una esfera socioeducativa.

Un currículo abierto, flexible y dinámico entrega la oportunidad de la inclusión del patrimonio cultural indígena dentro de su marco ideológico. La elasticidad del currículo potencia la capacidad de observar en la otredad nuevos caminos y temáticas de aprendizaje. En contextos en los cuales el currículo ha logrado integrar el patrimonio cultural de sus pueblos indígenas se ha asumido implícitamente el reconocimiento del valor de los sistemas de conocimientos y cosmovisión indígena (Sterenberg, 2013; Wang, 2015). No obstante, este proceso no ha ocurrido de manera uniforme ni tampoco consistentemente dentro de una o más dimensiones curriculares (Putnam et al., 2011). En consecuencia, se instala como una tarea pendiente.

\section{Metodología}

El presente estudio utilizó una metodología cualitativa de análisis de casos comparados, los cuales son fundamentalmente interpretativos, porque implican una relación teórico-metodológica entrecruzada con evidencia empírica (Jürgen, 2011). En sí mismo, el proceso de selección, a un nivel de diseño investigativo y metodológico, es parte de una instancia de interpretación, la que se propicia, por una parte, en la selección de casos y, por otra, en la selección de criterios de comparación. Para Jürgen (2011) los estudios de casos comparados proponen un plusvalor investigativo importante y distintivo, ya que a través de sus hallazgos es posible proyectar conclusiones adicionales tanto para el caso mismo, como para las interrelaciones entre estos. 
Según Maxwell (2005) la selección de los casos es un paso clave en los estudios de casos comparados, debido a que este tipo de estudios requiere diseñar unidades analíticas de comparación, lo cual significa crear criterios de comparación que atienden variables sostenidas dentro de los casos. En razón de ello, es clave hallar criterios que puedan ser efectivamente comparados dependiendo de la naturaleza del caso (Jürgen, 2011). Por consiguiente, una primera aproximación metodológica fue seleccionar los casos.

Australia, Chile y Sudáfrica son tres países ubicados en diferentes continentes. Pese a su remota conexión, los tres presentan elementos en común, específicamente en cuanto a la situación patrimonial de los pueblos indígenas dentro del currículo, que hace coherente su selección en términos de este estudio (Maxwell, 2005). Uno de estos elementos es de componente histórico, ya que los tres países fueron colonias europeas, por lo que terminó predominando una cultura hegemónica western por sobre lo previamente vivido, dentro de procesos homogeneizantes y totalizantes. Un segundo elemento en común se advierte en la necesidad política de establecer mecanismos de generación de identidad nacional. Una vez que estos países consiguieron la independencia política, se comenzaron a generar procesos de reestructuración e integración nacional que tuvieron como foco generar identidad a nivel país. Un tercer elemento tiene relación con la resistencia y marginación de las comunidades indígenas al momento de construir la nación. En los tres casos, se advierte una resistencia en distintas maneras respecto de lo que significa ser parte de una cultura nacional. Finalmente, un último elemento en común entre estos tres países se conecta con las constantes estrategias políticas/gubernamentales por integrar y revalorar el patrimonio cultural de los pueblos indígenas a nivel socioeducativo, al menos desde los discursos formales, nacionales y centralizados. En suma, Australia, Chile y Sudáfrica deben enfrentar una situación conflictiva con y ante los pueblos indígenas y su inclusión a nivel nacional y específicamente en el ámbito curricular.

Los estudios de casos comparados se caracterizan por elevar ciertas categorías de cruce relacional (Maxwell, 2005), las que se expresan en criterios de comparación que son entendidos como 
unidades de categorización selectiva (Eisenhardt \& Graebner, 2007). Dichos criterios son el punto inicial para la selección de casos y también para la relación entre los mismos. En este estudio, luego de la selección, se diseñaron y validaron criterios de comparación que incluyeron tres dimensiones: identidad, categorización curricular y conocimiento y aprendizaje indígena. Estas instancias fueron creadas de manera deductiva sobre la base de la información y de los datos contenidos en los marcos curriculares, entendidos como fuentes de información (Maxwell, 2005). Es importante comprender la primera dimensión de identidad porque tensiona dos mundos, el indígena y el no indígena, por medio de la aspiración a la construcción de una identidad nacional. En esta dimensión se compararon las ideas de nación, integración y sentido de pertenencia. La segunda dimensión, categorización curricular, es relevante puesto que permite razonar acerca de cómo se categoriza a las comunidades indígenas desde un referente curricular, es decir, cómo ha sido concebida la otredad, o bien, si no es considerada otredad, cuáles son los atributos, potencialidades y características específicas plasmadas en el currículo para categorizar a determinada comunidad. Finalmente, la dimensión conocimiento y aprendizaje indígena busca comparar qué saberes han sido relevados de manera positiva en y por el currículo a un nivel nacional.

Por último, las investigaciones comparadas en la actualidad son altamente pertinentes a causa del acaecimiento de lo transnacional en el campo de acción educacional, obligando a la generación de marcos conceptuales y teóricos próximos a realidades internacionales (Boeije 2010; Phillips \& Schweisfurth, 2007).

\section{Análisis de la información}

Las fuentes analizadas son tres referentes curriculares correspondientes a tres casos diferentes. Para el caso australiano, Cross-Curriculum Priorities: Aboriginal and Torres Strait Islander Histories and Cultures (2015); en el caso sudafricano, National Curriculum Statements: Grades R-12 (2013) y, finalmente, para el caso chileno se utilizó el Marco curricular lengua indígena $1^{\circ}$ a $6^{\circ}$ básico (2009). El referente curricular de Australia, Chile y Sudáfrica fue analizado a través de una 
comparación deductiva a un nivel discursivo desde tres dimensiones (Eisenhardt \& Graebner, 2007). El análisis de la información se encuadra en la posibilidad de responder preguntas determinadas por los criterios de comparación. De este modo, los tres criterios de comparación entendidos como dimensiones complejas sirven de parámetro para analizar discursivamente el currículo. No existiendo una única manera de generar análisis a partir de discursos (Phillips \& Schweisfurth, 2007), se optó por analizar, a un nivel discursivo, conceptos clave más que estructuras semióticas lineales. Esta línea de análisis está en concordancia con el análisis de discurso pragmático (ADP) propuesto por Potter y Wetherell (1987), puesto que lo importante es realizar preguntas al discurso centrándose en conceptos que en definitiva son ideología. Por lo mismo, fueron seleccionados los conceptos emergentes sobre la base de los criterios de comparación y no al revés. De ahí que dichos criterios sean definidos previamente y no a partir de categorías emergentes, ajustándose a un análisis de discurso pragmático (Boeije 2010; Phillips \& Schweisfurth, 2007; Potter y Wetherell, 1987).

Una de las consideraciones relevantes para analizar estudios de casos comparados es el procedimiento de análisis. Puesto que es importante seguir una organización lógica y coherente al momento de estudiar la información, fueron analizados cada uno de los referentes curriculares de manera individual para luego compararlos en función de los criterios. En coherencia con lo anterior, a continuación se presentan los casos estudiados.

\section{Australia, currículo y patrimonio cultural indígena}

Recientemente en 2015, Australia presentó un currículo más abierto y flexible incorporando el patrimonio cultural indígena no solo desde la disciplina de la historia, sino como una prioridad nacional en diversas líneas y áreas de aprendizaje. De acuerdo con lo establecido por el Ministerio de Educación Australiano (2015), el currículo exhibe estándares nacionales para mejorar los aprendizajes de todos los jóvenes australianos y frente a esta premisa, Australia ha reconocido una brecha importante entre los estudiantes indígenas y los no indígenas. Para cerrar esta brecha de 
resultados de aprendizaje, como fue expresado, es una prioridad nacional centrarse en dos objetivos:

- Estudiantes aborígenes tienen que ser capaces de verse a sí mismos, sus identidades y culturas deben estar reflejadas en el currículo de cada una de las áreas de aprendizaje, pudiendo plenamente participar en este y construir su autoestima.

- Las historias y culturas de los estudiantes aborígenes son una prioridad para diseñar atendiendo a todos los estudiantes, con la finalidad de generar reconciliación, respeto y reconocimiento de una de las más antiguas culturas que existen en el mundo actual (Cross-Cultural Curriculum: Aboriginal and Torres Strait Islander Histories and Cultures, 2015).

Dentro de esta prioridad nacional, como ha sido explícitamente escrita, se visualiza la existencia de un plan a nivel nacional de integración curricular. The Aboriginal and Torres Strait Islander Histories and Cultures se advierte como una prioridad nacional y eso es algo visto de manera positiva en términos de aprendizaje y reconciliación nacional, especialmente pensando en la fragmentación nacional (Nakata, 2011). Esto es importante, porque se advierte aquí la idea de prioridad en tono de urgencia, es decir, se establece una necesidad apremiante de incluir a los pueblos indígenas en un contexto actual valorando y reconciliándose con ellos.

En el contexto de aprendizaje, enmarcado en el currículo nacional de Australia, se establece el siguiente cuadro como marco conceptual para los estudiantes aborígenes. 


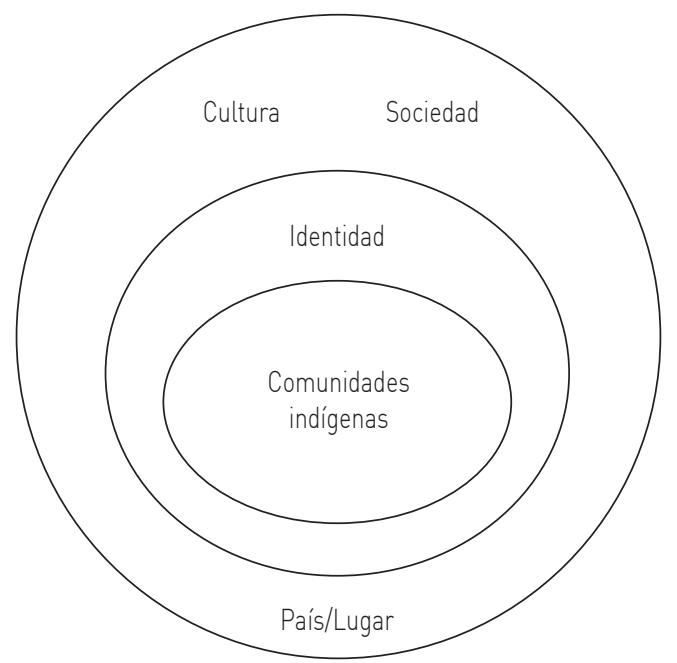

Figura 1. Conceptual framework for the Aboriginal and Torres Strait Islander Histories and Cultures priority (2015)

Fuente: Elaboración propia.

Según el Ministerio de Educación Australiano (2015) este marco conceptual propone como centro a las comunidades indígenas insertas en un contexto macrosocial. Al mismo tiempo, expresa una visión holística respecto del entendimiento del patrimonio cultural indígena en Australia. Sin embargo, el marco emerge con la finalidad de situar a estas comunidades en un contexto de aprendizaje, porque la conexión entre los estudiantes indígenas, la sociedad y la cultura se realiza mediante la identidad. Por consiguiente, la identidad se presenta como un elemento eje dentro del marco curricular australiano.

\section{Chile, currículo y patrimonio cultural indígena}

En Chile existe un currículo diseñado especialmente para los pueblos indígenas. Este currículo se encuentra enmarcado en un programa de modernización e inclusión educativa. Dicha idea tiene en su foco dos conceptos centrales que se despliegan de manera reiterada a lo largo del discurso: bilingüismo e interculturalidad. Según lo declarado por el Mineduc (2009) este programa de integración y valoración del patrimonio cultural indígena obedece a los requerimientos específicos engendrados por los pueblos indígenas y la sociedad nacional. 
Al unísono, la posibilidad de generar un programa de educación intercultural bilingüe atiende a la idea de propiciar procesos de mejora de la calidad y equidad de la educación (Hevia, 2005). En suma, bilingüismo e interculturalidad son dos conceptos clave en esta referencia curricular.

En el programa de Educación Intercultural Bilingüe (EIB) la idea de escuela y comunidad son latentes. Los estudiantes indígenas y su patrimonio cultural deben ser encausados en una escuela que potencie sus saberes. De este modo, los conocimientos y habilidades pueden ser desarrollados dentro de una escuela que cuenta con un proyecto educativo sostenido en una EIB (Cañulef y Galdames, 2002). La escuela como entidad social debe estar en coherencia con una EIB, ya que desde allí se generan adecuaciones curriculares que establecen la conexión necesaria entre currículo y contexto, la que en el caso chileno queda resuelta, según el Ministerio de Educación, en una descentralización del currículo, abriendo la posibilidad de autonomía para que las escuelas realicen adecuaciones contextuales que favorezcan el aprendizaje de los estudiantes indígenas.

A nivel curricular, existe una valoración lingüística y patrimonial de los pueblos indígenas. Esto queda expresado en la preocupación transversal por establecer la lengua indígena como primera o segunda lengua dentro de las escuelas que cuentan con una alta matrícula indígena. Uno de los focos prioritarios tiene relación con la "contribución para la recuperación, mantención y desarrollo de las lenguas indígenas en un contexto indígena" (Mineduc, 2009). Tal foco, presenta un elemento interesante, ya que sitúa el patrimonio cultural indígena desde la propia comunidad. Se percibe a nivel curricular la tendencia a asociar la preservación del patrimonio cultural indígena, en este caso lingüístico, en una interacción emplazada dentro de una microesfera. En otras palabras, una interacción dentro de una dimensión local. De allí que el enfoque sea lingüístico, puesto que la prioridad de aprendizaje propuesta en el currículo, es la necesidad de aprender la lengua de su pueblo con la finalidad de insertarse dentro de un contexto local, entablando relaciones sociales dentro de su comunidad próxima (Merino, 2006; Quilaqueo et al., 2010). 
Finalmente, en Chile se presenta un avance parcial en materia curricular referente a los pueblos indígenas y su patrimonio cultural, el cual también puede ser observado en un salto disciplinario, puesto que anteriormente el contenido curricular de los pueblos indígenas estaba encapsulado única y exclusivamente en la asignatura de Historia, Geografía y Ciencias Sociales. De forma que se abre la discusión hacia la interculturalidad como vía de educación en contextos indígenas, no solo en cuanto a contenidos disciplinarios, sino más bien a la construcción de comunidades de aprendizaje basadas en proyectos lingüísticos (García, 2007). Sin embargo, la sustentabilidad de este proyecto de EIB solamente se enfoca en niveles preescolares y básicos quedando pendiente la pregunta de por qué no avanzar curricularmente hacia los demás niveles o también por qué el currículo descansa en niveles medios y superiores en la disciplina de Historia.

\section{Sudáfrica, currículo y patrimonio cultural indígena}

Luego del apartheid en Sudáfrica, una de las primeras tareas fue redireccionar el sistema educativo a través del diseño e implementación de un nuevo currículo nacional. El sistema educativo post-apartheid fue pensado para integrar no solo a los pueblos indígenas, sino también y fundamentalmente para reconstruir y reconciliar a un país dramáticamente fraccionado (Msimanga \& Shizha, 2014; Simeon, 2011).

Sudáfrica es una sociedad hondamente heterogénea con once lenguas oficiales. La consideración de estas once lenguas se transformó en un desafío mayor para los diseñadores curriculares, porque ¿cómo hacer suficientemente pertinente y contextual los estándares nacionales para las onces lenguas oficiales? Desde este enfoque, se pensó en un currículo altamente integrador, capaz de asumir la diversidad como eje central. Pese a los múltiples avances, reconocidos internacionalmente, ello está lejos de ser logrado, puesto que aún prevalece una cultura blanca dominante por sobre los cuerpos de conocimiento de los pueblos indígenas (Murray, 2012; Royce, 2010). 
Según lo declarado por el Ministerio de Educación Sudafricano:

En el art. 108 de 1996, fundado nuestro Estado democrático y común ciudadanía sobre los valores de la dignidad humana, los logros de equidad y los avances en derechos humanos y libertades (Sección la), es que estos valores incluyen a todos para formar parte de una responsabilidad nacional y desafío de construir y cuidar una sociedad humanizada, no para unos pocos sino para todos los sudafricanos (Republic of South Africa, 2013).

En función de lo anterior, se desprende que la integración nacional será alcanzada a través de un proceso humanizante, siendo una tarea de y para todos. Los pueblos indígenas están sumidos en un proceso nacional que no hace diferencia entre blancos y negros, ni tampoco entre indígenas y no indígenas. La situación puntual en que se encuentran también se suma a la situación de otras entidades (étnico-raciales) que deben incorporarse a un proceso de integración nacional. De ahí que la situación en Sudáfrica en términos educativos y primordialmente curriculares sea aún más compleja.

Sudáfrica en los últimos años planteó un avance curricular a nivel nacional. Se implementó un nuevo currículo, planes de estudio y políticas de evaluación (Curriculum and Assessment Policy Statements, CAPS, 2012) dejando atrás el currículo nacional vigente del año 2005. El cambio fue suscitado debido a que existía escasa precisión acerca de los contenidos, habilidades y objetivos fundamentales, tanto en los niveles educativos como en las distintas disciplinas de aprendizaje (Royce, 2010; Simeon, 2011). Otro aspecto relevante fue que este currículo caminó en un recorrido más sensitivo y representativo (Chisholm, 2005). El proceso de desarrollo curricular pasó un currículo más prohibitivo e instalado en la negación a uno más sensitivo. Por ejemplo, expresiones tales como un currículo no racista, no sexista, no partidario (National Curriculum, 2005) en comparación con expresiones tales como; pluriétnico, representativo de razas, sexo, religión y orientaciones culturales y profesionales (National Curriculum Statements, NCS, 2013) grafican dicho transitar. El "no" en términos simbólicos representa prohibición, censura y negación. Suprimiendo este concepto se avanzó en una actitud discursivamente más 
inclusiva. Es un cambio léxico que representa un nuevo paradigma socioemocional ante la diversidad y su patrimonio cultural.

Hasta aquí el escenario de los pueblos indígenas ha quedado puesto a un mismo nivel de inclusión de otros pueblos o agrupaciones sociales que también han sido marginadas dentro del currículo sudafricano, lo cual se vislumbra por un contexto sociohistórico propio del devenir del país que declara cuatro principios establecidos por el Departamento de Educación Nacional (National Curriculum Statements, 2013):

- Derechos humanos.

- Inclusión.

- Desarrollo ambiental.

- Justicia social.

Realizando una aproximación más específica en el segundo principio de inclusión, puesto que es dentro de él donde se ciñe el patrimonio cultural indígena, es posible advertir que el currículo sudafricano presenta una valoración lingüística considerándola como patrimonio cultural indígena. No obstante, es una preocupación más metodológica que práctica. Esto puede ser explicado debido a que las preocupaciones curriculares están centradas en valorar el conocimiento, mantención y difusión de las lenguas indígenas dentro del mismo currículo, más que en la propia práctica pedagógica y en el área de aprendizaje (Murray, 2012). La explicación de ello se suscita porque el diseño y la estructura curricular son nuevos y buscan esencialmente desprenderse del símbolo western y colonial contenido en el precedente currículo nacional.

\section{Discusión}

La necesidad de introducir cambios para generar procesos de validación y reconocimiento del patrimonio cultural indígena se hace notorio en los tres casos presentados. Un proceso de inclusión e integración de los pueblos indígenas y su patrimonio ha sido posicionado como un desafío necesario de alcanzar en el siglo XXI (Abramovich, 2006). Existe una tendencia internacional por valorar el patrimonio cultural indígena desde una perspectiva curricular. 
A continuación se presenta una tabla que agrupa los tres casos estudiados: Australia, Chile y Sudáfrica, en correlación con las tres criterios de categorización expresados en dimensiones: dimensión identidad, dimensión categorización curricular y dimensión conocimiento y aprendizaje indígena²

Tabla 1

Patrimonio indígena y currículo en Australia, Chile y Sudáfrica

\begin{tabular}{|c|c|c|c|}
\hline & Australia & Chile & Sudáfrica \\
\hline $\begin{array}{l}\text { Dimensión } \\
\text { identidad }\end{array}$ & $\begin{array}{l}\text { Los estudiantes } \\
\text { indígenas participan } \\
\text { activamente en } \\
\text { el desarrollo } \\
\text { de la identidad } \\
\text { australiana. }\end{array}$ & $\begin{array}{l}\text { La identidad se construye desde un } \\
\text { enfoque lingüístico. La lengua es un } \\
\text { conocimiento esencial en la comprensión } \\
\text { y valoración de la cultura, por lo que } \\
\text { este sector enfatiza el aprendizaje de } \\
\text { la lengua y fomenta, a través de ella, } \\
\text { la aproximación a la cultura. Se asume } \\
\text { que desde el aprendizaje del idioma se } \\
\text { pueden aprender aspectos fundamentales } \\
\text { de una cultura, tales como la circularidad } \\
\text { del tiempo, la relación de parte a todo con } \\
\text { la naturaleza, la posición y definición de } \\
\text { la persona en relación con el entorno y } \\
\text { la armonía entre pares. Se comprenden } \\
\text { como prácticas lingüísticas asociadas a la } \\
\text { cultura, que forman parte del patrimonio } \\
\text { histórico de cada pueblo. }\end{array}$ & $\begin{array}{l}\text { En Sudáfrica es importante } \\
\text { valorar la identidad de } \\
\text { todas las comunidades } \\
\text { que forman parte del país. } \\
\text { Específicamente, el legado } \\
\text { lingüístico, cultural y de } \\
\text { conocimiento. }\end{array}$ \\
\hline $\begin{array}{l}\text { Dimensión } \\
\text { categorización } \\
\text { curricular }\end{array}$ & $\begin{array}{l}\text { Los estudiantes } \\
\text { indígenas son } \\
\text { fuertes, resilientes, } \\
\text { ricos y diversos. Los } \\
\text { dos grandes grupos } \\
\text { aborígenes, como } \\
\text { primeros habitantes } \\
\text { del lugar y país, } \\
\text { han demostrado } \\
\text { resiliencia ante un } \\
\text { proceso histórico y } \\
\text { los impactos de la } \\
\text { colonización. }\end{array}$ & $\begin{array}{l}\text { Los pueblos indígenas son diversos } \\
\text { y aportan a comprender el pasado y } \\
\text { presente de la nación. }\end{array}$ & $\begin{array}{l}\text { Los indígenas y su cultura } \\
\text { poseen una rica historia y } \\
\text { herencia en el país como } \\
\text { importantes contribuidores } \\
\text { de la formación de valores } \\
\text { fundamentales contenidos } \\
\text { en la constitución de la } \\
\text { República de Sudáfrica. }\end{array}$ \\
\hline
\end{tabular}

2 Cabe señalar que los escritos aquí presentes son extractos traducidos del marco curricular de cada país. 


\begin{tabular}{|c|c|c|c|}
\hline $\begin{array}{l}\text { Dimensión } \\
\text { conocimiento } \\
\text { y aprendizaje } \\
\text { indígena }\end{array}$ & $\begin{array}{l}\text { Aboriginal and } \\
\text { Torres Strait } \\
\text { Islander poseen } \\
\text { sofisticados } \\
\text { sistemas de } \\
\text { creencias } \\
\text { espirituales e } \\
\text { intelectuales } \\
\text { basadas en la tierra, } \\
\text { el mar, el cielo y } \\
\text { los cursos de agua. } \\
\text { Las formas de vida } \\
\text { son únicamente } \\
\text { expresadas a través } \\
\text { de las vías del ser, } \\
\text { conocer, pensary } \\
\text { hacer. }\end{array}$ & $\begin{array}{l}\text { El currículo organiza la enseñanza y } \\
\text { el aprendizaje de la lengua desde sus } \\
\text { características semánticas, entendiendo } \\
\text { que la construcción de un idioma } \\
\text { se relaciona directamente con las } \\
\text { significaciones culturales del pueblo } \\
\text { que la utiliza y, asimismo, tiene como } \\
\text { propósito formativo la enseñanza y } \\
\text { el aprendizaje de la lengua indígena, } \\
\text { principalmente en los establecimientos } \\
\text { con matrícula escolar indígena. }\end{array}$ & $\begin{array}{l}\text { El aprendizaje acerca de la } \\
\text { naturaleza del espíritu en } \\
\text { relación con el ambiente y la } \\
\text { comunidad es considerado } \\
\text { central para comprender el } \\
\text { significado de la vida. } \\
\text { Se presenta una interesante } \\
\text { valoración del aporte } \\
\text { científico de las comunidades } \\
\text { indígenas y su diálogo } \\
\text { abierto con la ciencia } \\
\text { tradicional. } \\
\text { Apropiado uso de la } \\
\text { tecnología de subsistencia } \\
\text { y crecimiento económico a } \\
\text { través de la utilización de los } \\
\text { sistemas de conocimiento } \\
\text { indígena. }\end{array}$ \\
\hline
\end{tabular}

Fuente: Elaboración propia.

En la primera dimensión de identidad, los tres casos presentan diferentes conceptos, aunque Sudáfrica y Chile comparten un enfoque identitario situado desde lo lingüístico. Esto puede ser explicado porque para Australia, de acuerdo con lo expresado por el referente curricular, existen únicamente dos grandes grupos indígenas: Aboriginal y Torres Strait Islander. En oposición, Chile reconoce ocho lenguas distintas y en Sudáfrica la situación es más amplia, con 11 lenguas oficiales. Es decir, para Australia la agrupación de los pueblos tiene relación con elementos no necesariamente lingüísticos, donde la apuesta pasa por una integración generalizada de los estudiantes indígenas de un territorio, como elementos diferenciados, por lo cual la cantidad de lenguas reconocidas hace prevalencia del concepto de diversidad cultural. En este sentido, se entiende que el aprendizaje de la lengua es fundamental en el proceso de construcción de identidad. El lenguaje entrega la posibilidad única de entender el mundo en el que vivimos, así como también es un importante factor que contribuye al sentido de pertenencia a un pueblo.

En Chile, a diferencia del caso sudafricano, se utiliza el mismo lenguaje como vía de identidad y aprendizaje. A modo de graficar lo anterior, el programa de educación intercultural bilingüe en Chile busca, "a través de los artículos 28 y 32, el respeto y promoción de las culturas indígenas, para lo cual establece: el uso y conservación de los idiomas indígenas, junto al español en las áreas de alta densidad 
indígena" (Mineduc, 2009). En el caso chileno, la lengua se manifiesta como patrimonio cultural intangible, que es una primera entrada a la construcción de la identidad. La lengua del pueblo indígena es patrimonio fundante y generador de las relaciones sociales dentro de la comunidad. Esta mirada está centrada en procesos de construcción identitaria desde una doble entrada: social e individualmente. En contraste, la situación en Sudáfrica -que si bien comparte una aproximación lingüística-, no presenta un plan de bilingüismo. Es más, la crítica constante se sitúa en que existe una contradicción lingüística, puesto que se valoran las lenguas indígenas, sin embargo, los idiomas de enseñanza y aprendizaje son afrikans e inglés (Murray, 2012). Tanto Australia como Sudáfrica, en tanto, presentan una postura de identidad más intercultural, a diferencia de Chile, que sitúa el proceso de identidad desde al aprendizaje de la lengua en la sociedad de origen. De ahí que adquiera sentido que el programa EIB se sustente en comunidades educativas con alta matrícula indígena.

En lo concreto, Chile posee un currículo separatista, es decir, un currículo ajeno, nuevo y exclusivo para escuelas con alto índice de estudiantes indígenas, que si bien es voluntario de implementar, representa una realidad diferente a Sudáfrica y Australia, países que han optado de forma consciente e intencional por integrar el patrimonio cultural indígena de manera transversal dentro de un marco curricular nacional.

Finalmente, en esta dimensión solo para Australia el proceso identitario es una prioridad nacional. Se visualiza constantemente a nivel discursivo la idea de prioridad, que hace referencia a un sentido de urgencia por instalar procesos de apropiación de la propia cultura indígena. Además, aquello se representa en el marco conceptual propuesto a nivel curricular donde se ubica el concepto de identidad en el corazón del plan.

Por su parte, la segunda dimensión, categorización curricular, plantea una demanda cognitiva, conceptual e ideológica interesante de ser analizada bajo el prisma de los diseñadores curriculares. La categorización curricular hace referencia a cómo se definen los pueblos indígenas dentro del currículo. Esto es importante ya que conjetura 
una aproximación clara y simbólica acerca de quiénes forman parte del patrimonio cultural indígena. En palabras más políticas, a quién pertenece el patrimonio cultural. En el caso australiano, a diferencia del chileno y sudafricano, aparece explícitamente definido cómo son los pueblos indígenas. Resulta fácil entender que para Australia son pueblos fuertes, resilientes, ricos y diversos, características que son puestas como cualidades inherentes a los pueblos indígenas de Australia a consecuencia de que han sabido sortear los obstáculos históricos dejados por la colonización. Su alto grado de resiliencia pareciera fomentar un respeto nacional ante las adversidades afrontadas por los aborígenes. En la misma línea, esta idea de respeto está en coherencia con la idea de incrementar la autoestima de los estudiantes indígenas en el contexto australiano. Se piensa que los efectos de esta valoración generarán impactos en la construcción de un autoconcepto de los estudiantes indígenas y su patrimonio cultural.

Otro elemento interesante de analizar en el caso australiano se conecta con la proposición de pueblo fundante. Los pueblos indígenas en Australia son fundantes del lugar y país, por la razón de su asentamiento temprano dentro del territorio nacional. Esta proposición manifiesta una actitud positiva ante los pueblos indígenas, como una cultura precedente que debe ser valorada. Asimismo, es un léxico que asume que el patrimonio de los pueblos no solo pertenece a ellos, sino que también a los australianos, puesto que comparten un lugar y país comunes. Por lo tanto, no solo es valorado el patrimonio cultural de los pueblos indígenas, sino que también ellos en sí mismos como comunidades son consideradas patrimonio cultural australiano.

Siguiendo la línea de pensamiento anterior, la categorización curricular en Sudáfrica también se encuentra aparejada con la idea de pueblo fundante. Sin embargo, en este caso está asociada con una formación valórica fundamental de los contenidos de la constitución sudafricana, de modo que es un pueblo fundante en una arena más político-constitucional, lo que demuestra que los pueblos indígenas han sido formadores de la nación con sus principios, ideas e historias. Esto apremia la necesidad de integrarlos no solo 
en decisiones político-educativas, sino también en una unión más concreta respecto de los propios contenidos curriculares que se deben diseñar e implementar en las escuelas.

Por otro lado, dentro del caso sudafricano y chileno es más difícil encontrar una categorización curricular explícitamente definida acerca de lo que significa ser un pueblo indígena, lo cual presenta una dualidad en relación con el patrimonio cultural, puesto que este se encuentra bien definido, en ambos casos, pero las comunidades que lo conforman no lo están. Es una definición que atiende al qué y no al quiénes, por lo cual es substancial, establecer una definición clara y concisa respecto de las características de los pueblos indígenas (Simeon, 2011), específicamente en el currículo, ya que de este modo se pueden potenciar elementos propios de la cultura en instancias educativas, sirviendo como orientación pedagógica a un nivel práctico tanto en los contenidos como en los objetivos de aprendizaje.

Australia, Chile y Sudáfrica conciben la categorización curricular desde un referente patrimonial e histórico. Es decir, desde una proyección en el presente, anclada en un pasado, que permite aglutinar herencias y legados culturales, tales como los modos de vivir, el lenguaje, costumbres, entre otros (Abramovich, 2006). Es necesario, por lo tanto, establecer una categorización de la comunidad indígena, así como también del patrimonio cultural. Pareciera ser más fácil, o una preocupación más notoria, establecer categorizaciones en torno al patrimonio cultural indígena en vez de categorizaciones correspondientes a la propia comunidad indígena.

Por último, la tercera dimensión, de conocimiento y aprendizaje indígena, plantea ideas acerca de cómo es valorado el patrimonio cultural indígena en términos de sistemas de conocimiento. A diferencia de las otras dimensiones, esta presenta menos elementos en común. El caso de Sudáfrica ha sido estudiado largamente en función del aporte de las ciencias indígenas en correspondencia con las ciencias tradicionales. Incluso, el Departamento de Educación ha sido clave en integrar elementos no tradicionales en las ciencias dentro del currículo con un alto impacto en las comunidades de aprendizaje (Msimanga \& Shizha, 2014). En este sentido, Sudáfrica 
ha sido capaz de presentar resultados de impacto ante la utilización del referente de conocimiento y aprendizaje propiciado dentro de las comunidades indígenas. Así, se han utilizado mecanismos desde los contenidos procedimentales extraídos de métodos de curación de enfermedades, reconocimiento de factores del clima, sistemas de sostenibilidad y viabilidad de asentamientos, entre otros (Nakata, 2011). Esto no es posible observar en los casos de Chile y Australia, de modo que es interesante reconocer los aportes, pero más aún exhibir resultados de los mismos en actividades diarias dentro de los procesos de enseñanza-aprendizaje, experimentados por los estudiantes indígenas y no indígenas, como lo demuestra el caso sudafricano.

En esta dimensión de conocimiento y aprendizaje, el enfoque australiano comienza desde una aproximación filosófica. Es decir, desde el entendimiento del mundo, de los significados y códigos que envuelven a un sujeto, al ser, al "yo". Esto es relevante porque sintoniza con las formas de aprendizaje generadas dentro y por las mismas comunidades indígenas. A diferencia de ello, en el caso chileno se entiende el aprendizaje no en términos de concepción filosófica, sino más bien enraizado en procesos de conocimiento tácito, esencialmente conectado con el aprendizaje de la lengua materna: a partir del aprendizaje y conocimiento de la lengua indígena, como patrimonio cultural, es posible aprender y relacionar elementos del diario vivir con los aprendizajes fecundados en la escuela (Merino, 2006).

La dimensión espiritual y ambiental ha sido tradicionalmente asociada al patrimonio cultural indígena. Tanto Australia como Sudáfrica se han encargado de posicionar dichas dimensiones dentro de sus referentes curriculares. Aquí el aprendizaje y conocimiento no solo se conjeturan en lo procedimental y/o declarativo, sino que también pueden ser vistos desde un axioma metafísico que potencia la capacidad de reconocerse uno mismo como sujeto en acción dentro de un ambiente con características particulares. De ahí que cobre importancia la naturaleza del espíritu y el significado de la vida. Este conocimiento introspectivo es reconocido explícitamente en el currículo nacional sudafricano. También se conecta con la idea de 
entender el ser, es saber, el pensar y el hacer propuesto por el referente australiano. Lo anterior plantea una dimensión más compleja de los procesos de aprendizaje, que advierte la necesidad de anclar los aprendizajes no solo en la exterioridad del ser, mezclando lo nuevo con lo precedente, lo cual es un desafío interior que problematiza la relación del "yo" y el ambiente. Fundamentalmente, se agrega la construcción del sujeto actuante en una realidad cambiante de aprendizaje, que moviliza una discusión curricular respecto de lo que es verdaderamente importante en la escuela.

\section{Conclusión, implicancias y limitantes}

En los últimos años Australia, Sudáfrica y Chile se han preocupado de instalar procesos de mejora curricular, valorando su patrimonio cultural indígena. Se exhiben referentes curriculares más flexibles que, dada su elasticidad, potencian la posibilidad de incluir, proteger y restaurar diferentes ideas provenientes del mundo indígena. Pareciera existir un currículo intercultural, no obstante es solo un incipiente diálogo cultural que no necesariamente involucra un poder compartido en materia curricular.

Los conceptos de identidad, lengua y patrimonio cultural se encuentran presentes en los tres casos estudiados. Pareciera ser que existe una deuda histórica para con los pueblos indígenas, por lo que se hace una valoración explícita de su patrimonio cultural. Por lo mismo, es posible advertir un cargo de conciencia histórica en torno a los dramáticos procesos vividos por estos, de modo que una manera de retribuir y realzar su patrimonio cultural es mediante la reposición de su legado dentro del currículo (García, 2007), lo que podría ser denominado como medidas de compensación curricular.

El análisis aquí presentado se basa en conceptos clave para el entendimiento del discurso. Así, identidad, cultura, patrimonio, historia, lengua, son algunos que dan luces de procesos de integración curricular. Sin embargo, dos conceptos parecen ser cruciales, uno de ellos tiene relación con la resiliencia y otro está conectado con el conocimiento. El caso australiano menciona la resiliencia explícitamente, donde este concepto pretende demostrar 
al país entero que los pueblos indígenas han sido capaces de levantarse ante procesos de violencia y conflicto que podrían haber atentado directamente contra la existencia de su comunidad. De esta forma, se recalca la capacidad de resistencia ante eventualidades complejas, la que se resalta como un valor eje dentro del currículo australiano, que podría ser proyectado y revisado en otros contextos curriculares. Por otro lado, el concepto de conocimiento plantea una discusión interesante, puesto que se valida el "no tradicional", tan legitimado como el patrimonio de propiedad intelectual dentro de las comunidades indígenas. Así, se amplía la visión de aprendizaje formal dentro de sistemas educativos tradicionales, lo que potencia la capacidad de adoptar el conocimiento a situaciones contextualizadas.

La pregunta central aquí es cómo avanzar en términos curriculares en relación con el patrimonio cultural indígena, o bien, de qué manera se puede estructurar un diseño curricular sustentado en la integración del conocimiento indígena. Pareciera ser que la respuesta australiana se aproxima más a la idea de una escuela inclusiva. Esto, en razón de que presenta un currículo intercultural entendido como prioridad nacional, con conceptos clave de cómo atender a la comunidad indígena, además de un claro referente pedagógico/práctico en relación a cómo abordar el patrimonio cultural indígena en cada subsector o área de aprendizaje. De ahí que en las orientaciones curriculares no solo deba ser importante qué aprender, sino también cómo aprender.

Otro aspecto importante se vincula con el cómo se construye, desde los referentes políticos, un currículo con integración del patrimonio cultural indígena. En esta acción, Chile presenta un currículo construido con los actores de la comunidad. Desde su fundación conceptual y estructural los actores son protagonistas del proceso, se encuentran envueltos dentro de las decisiones curriculares, lo cual representa una invitación interesante que potencia la capacidad de generar aprendizajes significativos y contextuales tanto en estudiantes indígenas como no indígenas. De igual modo, pareciera ser un proceso de más larga duración, pero que podría tener un impacto mayor en las comunidades indígenas. 
En los tres casos analizados existe una opción curricular dominada por una cultura hegemónica, lo que Quintrineo y McGinity (2009) han denominado como un currículo monocultural. Es decir, una cultura se impone por sobre las otras, donde pese a coexistir lineamientos y conocimientos desde el mundo indígena, existe una clara tendencia a incluir elementos, pero no a agenciar cambios para posicionarlos desde un nivel no opresivo.

En los tres casos presentados se despliegan limitantes. Primero, son temas que responden a contextos específicos y situaciones históricas particulares. Si bien la metodología asegura la coherencia del análisis, las conclusiones no son generalizables y deben ser revisadas considerando un espacio y tiempo histórico concreto. En otro plano, los referentes curriculares son de diferente naturaleza. En el caso chileno, a diferencia de Australia y Sudáfrica, se presenta un currículo especialmente para la comunidad indígena. Lo que en definitiva, podría generar ciertas complejidades al momento de entender el concepto de inclusión. Por lo tanto, se abre un espacio de indagación interesante en la proyección de este estudio y su correlación en textos de estudio y/o materiales didácticos entregados por los referentes ministeriales.

En última instancia, se advierten de manera positiva los procesos de generación de diseños curriculares más acordes con los tiempos de inclusión. La existencia de un discurso proclive de aceptación a la diversidad cultural se ha instalado como principio. En los referentes curriculares la diversidad cultural se ha institucionalizado. El desafío se emplaza en la capacidad de generar estudiantes, profesores y directivos capaces de leer el contexto de aprendizaje, porque un primer paso es apreciar el patrimonio cultural indígena, pero la finalidad curricular es integrar dicho patrimonio a la vida cotidiana de los estudiantes.

\section{Referencias}

Abramovich, V. (2006). Una aproximación al enfoque de derechos en las estrategias y políticas de desarrollo. Revista CEPAL, 88, 35-50. Recuperado de: http://www.cepal.org/es/publicaciones/11102aproximacion-al-enfoque-derechos-estrategias-politicas-desarrollo 
Ball, S. (2012). Global education Inc. New policy networks and the neo-liberal imaginary. London: Routledge.

Birch, E. (2010). Indigenous perspectives in the Australian curriculum. TLN Journal, 17(2), 16-18.

Boeije, H. (2010). Analysis in qualitative research. Los Angeles: Sage.

Cañulef, E. y Galdames, V. (2002). Algunas orientaciones curriculares para la educación intercultural bilingüe en contextos mapuches. Santiago de Chile: Mineduc.

Carnoy, M. (1999). Globalization and educational reform: What planners need to know. Fundamentals of Educational Planning. Paris: Unesco.

Chisholm, L. (2005). The making of South Africa's National Curriculum Statement. Journal of Curriculum Studies, 37(2), 193-208. https://doi.org/10.1080/0022027042000236163

Coombe, R. J. (2001). The recognition of indigenous peoples' and community traditional knowledge in international law. St. Thomas Law Review, $14,275-285$.

CAPS. Curriculum and Assessment Policy Statements, CAPS, (2012). Recuperado de: http://www.education.gov.za/Portals/0/CD/ National\%20Curriculum\%20Statements\%20and\%20Vocational/ CAPS $\% 20$ SP \% $20 \% 20$ MA T HEMA T ICS \% 20 G R \% 207 - 9. pdf?ver=2015-01-27-160141-373

Eisenhardt, K. \& Graebner, M. (2007). Theory building from cases: Opportunities and challenges. Academy of Management Journal, 50(1), 25-32. https://doi.org/10.5465/amj.2007.24160888

García, Z. (2007). Estrategias educativas para la valoración del patrimonio cultural en la educación básica en Venezuela. Educacere, 11(39), 136147. Recuperado de: http://www.scielo.org.ve/scielo.php?script=sci_ arttext\&pid=S1316-49102007000400012\&lng=es\&tlng=es

Geertz, C. (2000). Culture, customs and ethics. Oxford: Polity.

Henry, M. (2010). Working with/against globalization in education. Journal of Educational Policy, 14(1), 85-97. https://doi.org/10.1080/026809399286512

Hevia, R. (2005). Políticas educativas de atención a la diversidad cultural. Brasil, Chile, Colombia, México y Perú. Santiago: Orealc-Unesco.

Jürgen, S. (2011). Sistema mundial y redes de interrelación: la internacionalización de la educación y el papel de la investigación comparada. En M. Caruso y H-E. Tenorth (Eds.), Internacionalización. Políticas educativas y reflexión pedagógica en un medio global (pp. 41106). Buenos Aires: Granica. 
Kuprecht, K. (2012). The concept of 'Cultural Affiliation' In NAGPRA: Its potential and limits in the global protection of indigenous cultural property rights. International Journal of Cultural Property, 19(1), 33-63. https://doi.org/10.1017/s0940739112000057

Leguina, J. y Baquedano, E. (2000). Un futuro para la memoria. Sobre la administración y el disfrute del patrimonio histórico español. Madrid: Visor.

Marginson, S. (2010). After globalization: Emerging politics education. Journal of Educational Policy, 14(1), 19-31. https://doi.org/10.1080/026809399286477

Maxwell, J. A. (2005). Qualitative research design: An interactive approach. Thousand Oaks, CA: Sage.

Mcmillan, F. (2013). The protection of cultural heritage: Common heritage of humankind, national cultural 'patrimony' or private property? Northern Ireland Legal Quarterly, 64(3), 351-364. Recuperado de: http://eprints.bbk.ac.uk/7289/1/7289.pdf

Merino, M. E. (2006). Discriminación percibida en el discurso de mapuches y sus efectos psicosociales. Análisis del discurso de mapuches residentes en Temuco y Santiago. (No 1051047). Chile: Fondo Nacional de Desarrollo Científico y Tecnológico.

Ministerio de Educación de Chile, Mineduc. (2009). Marco curricular lengua indígena $1^{\circ}$ a $6^{\circ}$ básico. Recuperado de: http://educrea.cl/marcocurricular-lengua-indigena-1-a-6-basico/

Msimanga, A. \& Shizha, E. (2014). Indigenous knowledge and science education in South Africa: What messages from the curriculum. Remapping Africa in the global space: Propositions for change. Brantford: Sense Publishers.

Murray, S. (2012). The challenges of designing a common, standards-based curriculum for all South Africa's languages. Per Linguam. A Journal for Language Learning, 28(2), 84-94. https://doi.org/10.5785/28-2-532

Nakata, M. (2011). Pathways for indigenous education in the Australian curriculum framework. Australian Journal of Indigenous Education, 40, 1-8. https://doi.org/10.1375/ajie.40.1

Phillips, D. \& Schweisfurth, M. (2007). Comparative and international education: An introduction to theory, method and practice. London: Continuum.

Potter, J. \& Wetherell, M. (1987). Discourse and social psychology: Beyond attitudes and behaviour. London: Sage.

Putnam, J., David, E. Bernard, J., \& Jerome, R. (2011). Cross-cultural collaboration for locally developed indigenous curriculum. 
International Journal of Multicultural Education, 13(2), 1-18. https://doi.org/10.18251/ijme.v13i2.400

Quilaqueo, D., Fernández, A., y Quintriqueo, S. (2010). Interculturalidad en contexto mapuche. Neuquén: Educo.

Quintrineo, S. y McGinity, M. (2009). Implicancias de un modelo curricular mono-cultural en la construcción de la identidad sociocultural de alumnos/as mapuches de la IX región de La Araucanía, Chile. Estudios pedagógicos, 35(2), 173-188. https://doi.org/10.4067/s071807052009000200010

Republic of South Africa, National Curriculum Guidelines (2015). Recuperado de: http://www.education.gov.za/Portals/0/CD/LPGs2007/LPG\%20 RELIGION\%20STUDIES.pdf?ver=2007-11-30-091510-000

Republic of South Africa. National Curriculum Statements: Grades R-12. (2013). Recuperado de: http://www.australiancurriculum.edu.au/ crosscurriculumpriorities/aboriginal-and-torres-strait-islanderhistories-and-cultures/in-the-learning-areas

Rizvi, F. \& Lingard, B. (2010). Globalizing education Policy. Abingdon: Routledge.

Royce, B. (2010). Indigenous knowledge as culturally centred education in South Africa. Africa Education Review, 7(1), 34-50. https://doi.org/10.1080/18146627.2010.485804

Rogers, C. (2012). Hearing the story: Critical indigenous curriculum inquiry and primary source representation in social studies education. Theory E Research in Social Education, 40(4), 339-370. https://doi.org/10.1080/00933104.2012.723242

Scott, D. (2008). Critical essays on major curriculum theorists. New York: Routledge.

Simeon, M. (2011). The absence of a home curriculum in post-apartheid education in South Africa. International Journal of African Renaissance Studies - Multi-, Inter and Transdisciplinarity, 6(2), 100-117. https://doi.org/10.1080/18186874.2011.650852

Simpson, T. (1997). Patrimonio indígena y autodeterminación. IWGIA: Copenhagen.

Sterenberg, G. (2013). Considering indigenous knowledges and mathematics curriculum. Canadian Journal of Science, 13(1), 18-32. https://doi.org/10.1080/14926156.2013.758325

The Australian Curriculum. (2015). Cross-Cultural Curriculum: Aboriginal and Torres Strait Islander Histories and Cultures. Recuperado de: http://www. 
192 ANÁLISIS COMPARADO SOBRE PATRIMONIO CULTURAL INDÍGENA Y CURRÍCULO: AUSTRALIA, CHILE Y SUDÁFRICA - F. Aravena

australiancurriculum.edu.au/crosscurriculumpriorities/aboriginal-andtorres-strait-islander-histories-and-cultures/overview

Wang, C. (2015). The review of multicultural curriculum transformation on indigenous ethnic curriculum policy development. Journal of Curriculum Studies, 10(1), 13-34.

Recibido: 30/06/2016

Aceptado: 05/12/2016 